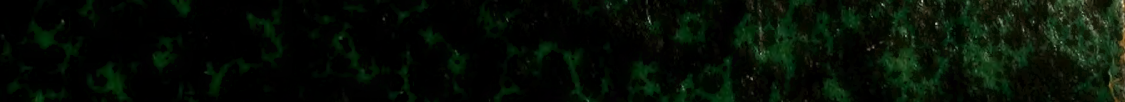

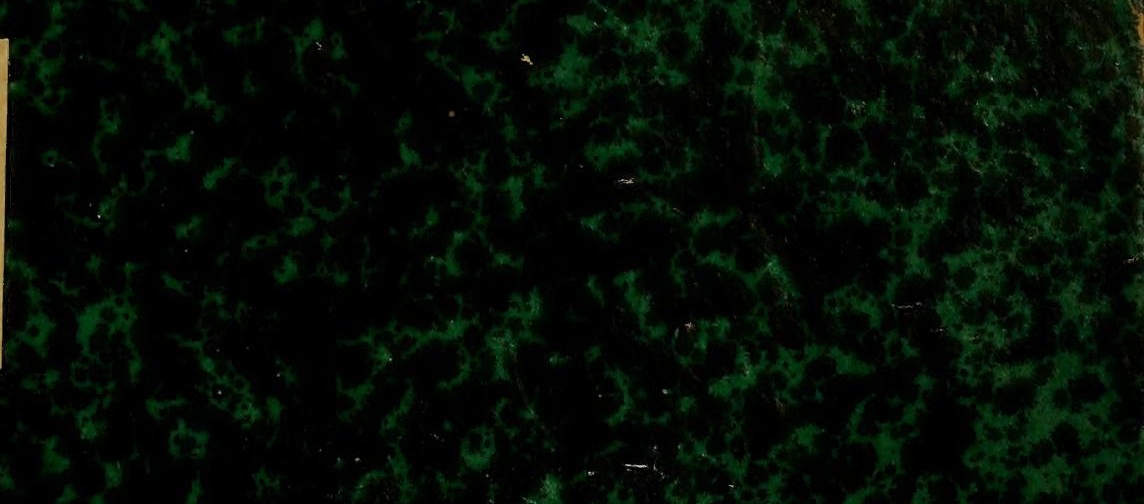

E ats

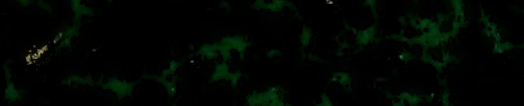
the

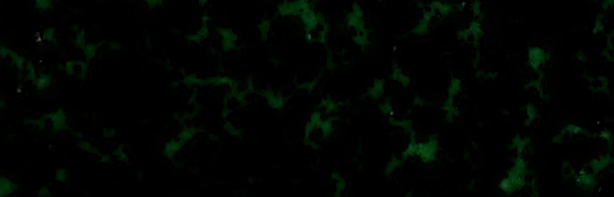

2. $x+2, x$

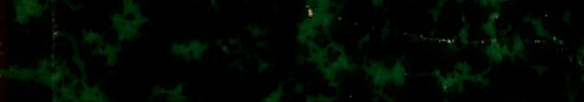

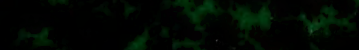

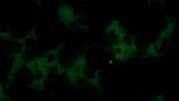<smiles>C1CCC2CCCC2CC1</smiles>
$\operatorname{lin}^{2}+2 \times 19$

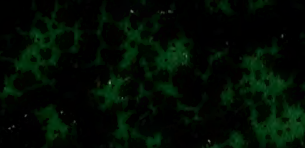

$\sin$ 


\section{HARVARD UNIVERSITY.}

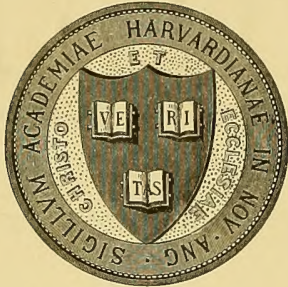

\section{LIBRARY}

OF THE

MUSEUM OF COMPARATIVE ZOÖLOGY 42,052

BEQUEST OF

WILIIAM MCM. WOODWORTH.

Tebruany 24,1915 


1859. VII.

MEDIC. $\mathbf{X}$.

\title{
DESCRIPTIO ANATOMICA
}

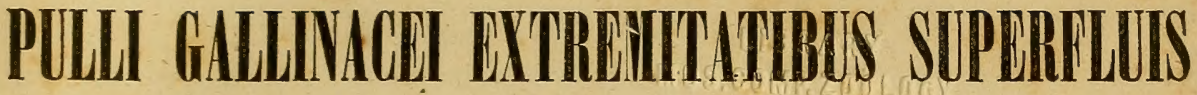 PRABDITI}

ssume cum

\section{DISQUUISITIONE PHISSIOLOGICA IIE ORTU NONSTRORLIII DLPLICILII PARISITICORIIII}

\section{DISSERTÁTIO INAUgURALIS ANATOMICO-PHYSIOLLGICA} QUAM

CONSENSU AET AUCTORITATE GRATIOSISSIMI MEDICORUM ORDINIS IN ACADEMIA CHRISTIANA-ALBERTINA PBO SUMMLS IN MEDICINA ET CHIRURGIA HONORIBUS RITE IMPETRANDIS

SCRIPSIT

\section{HENRICUS FREDERICUS MÜLLER}

\author{
LENSAHNENSIS.
}

ACGEDUNT TABULAE II LITHOGRAPHICAR

\author{
KILIAE. \\ EXOFFICINA C. F. MO H R. \\ MDCCCLIX.
}




\section{.IIV eter I}

'. . .

\section{ADTMOTAKA OTTETSDERT}

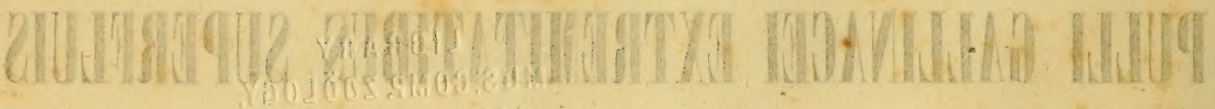

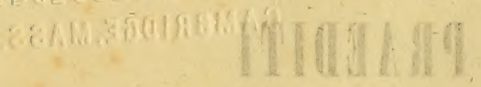

His? nithe

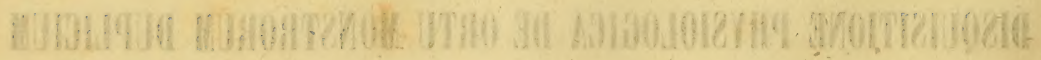

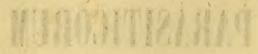

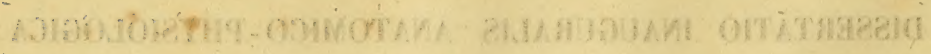
i. $x$ is

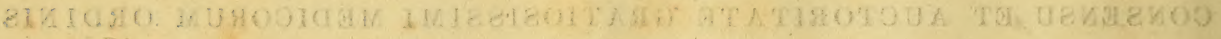

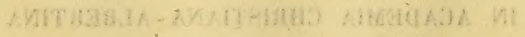

zuका elgrentiout an:

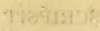

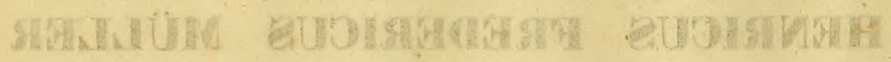

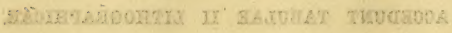

\section{Imprimatur.}

74hath

Dr. P. L. Panum,

h. t. Decanus. 
Monstrorum avium, quae in collectionibus anatomicis et descriptionibus omnino minus numerosa exstant, ea, quae in mammalibus et hominibus rariora inveniuntur monstra quibus extremitates superfluae suspensae sunt, pro numero mirum in modum crebriora occurrunt. Ita apud C. Chr. Heusnerum (descript. monstror. avium, amphibior., piscium, quae exstant in museo universitatis litterarum Berolinensis, eorumque cum monstris mammalium comparatio, dissertat. inaug. Berol. 1824. 8.) triginta trium avium monstrorum quindecim extremitatibus superfluis instructa sunt, quorum pulli gallinacei undecim, anates duae, anseres duo; apud G. Otto (monstror. sexcentor. descriptio anatomica Vratislav. 1841, fol.) quinquaginta duorum avium monstrorum undetriginta: pulli gallinacei quattuordecim., anates quinque, anseres quinque, columbae quattuor, fulica una; apud Gurltium (Catalog des zootomischen Museum's der Königl. Thierarzneischule bis Ende 1837, nach dem Magazin für die gesammte Thierheilkunde 4. Jahrgang, 2. Stück.) tredecim avium monstrorum, sex: pullus gallinaceus unus, anates duae, anseres duo, columba una; apud D'Alton (de monstris quibus extremitates superfluae suspensae sunt, commentatio Halis 1853.) triginta trium avium monstrorum duplicium, quae e collectione anatomica Halensi afferuntur, viginti duo extremitatibus superfluis praedita, pulli gallinacei septem, anseres septem, anates tres, columbae duae inveniuntur. Deinde, teste professore Panum, in museo Meckelii (secundum Bendzii catalogum) anno 1833, viginti septem avium monstrorum nostri generis erant viginti: quorum pulli gallinacei septem, anates sex, anseres quattuor, pavo unus, columba una, fringilla Canaria una; in museis Hafniae quattuordecim avium monstrorum, quinque, extremitatibus superfluis instructa, asservantur. In museo anatomico Kiliensi undecim avium monstrorum septem illius generis exstant. Denique J. T. Schneider, ${ }^{1}$ ) tamen minus accurate atque diligenter gallum extremitatibus superfluis instruetum descripsit: in quo gallo, qui per sex aut septem menses vixerat, pelvis irrperfecta cum osse sacro, extremitatesque superfluae direetione normalibus oppositae se ostendebant.

1) Beschreibung eines 4 füssigen Haushahns mit einer Abbildung. Grörlitz 1827. 
Praeterea de illius generis monstris multa afferuntur a Meyer, Alderovando, Haller, Poli, Kundmann, Reaumur, Regnault, Hoffmann, de Westhoven, Lidbeck, Rommel, Wolff, Fougeroux, Valisneri.

Exsistit autem quaestio difficilis, quomodo monstra illa orta sint. De qua re hae duae vigent sententiae sibi contrariae: altera monstra nostra ex uno, altera ex duplici germine oriri vult. Inter illius sententiae sectatores J. F. Meckel omnia monstra duplicia e primarie simplici germine nimia vi plastica enasci sumit; quin hanc nimiam vim plasticam cum illa vi luxuriante, qua regenerentur polyporum partes resectae, comparat. Idemque Leuckart primordia illorum monstrorum simplicia esse, voluit, e quorum aut minore aut majore fissione plus minus perfecta monstra duplicia exorirentur. Quae Leuckarti sententia in is casibus valere nobis videtur, in quibus, ut exemplo utar, duae tibiae cum uno femore, duo aut plures pedes cum una tibia conjuncti, aut digiti superflui exsistunt. Hic enim non nobis difficile videtur cogitatione comprehendere, primordia illorum organorum simplicia materiae abundantia (scilicet priusquam formentur elementa histologica) findi itaque duplicia reddi posse. Sed ad ea, quae in continuo blasdotermate evolvuntur organa, illa theoria adhiberi posse non nobis videtur; quae enim, si finderentur et idem blastoderma findi oporteret; quo facto, quin evolutio omnino tolleretur, haud dubium esset; neque jam fissionis causae facile perspicerentur. Itaque fissionis theoria non nobis adhiberi posse videtur ad eos casus, in quibus praeter extremitates superfluas pars columnae vertebrarum secundae occurrit; sed etiam, si haec secundae columnae vertebtarum vestigia desunt, in illis casibus, in quibus solae extremitates snperfluae cum normalibus non conjunctae, sed locis longe diversis suspensae se ostendunt, nihil valere videtur.

Neque jam minus de altera sententia ortus monstrorum duplicium e duobus germinibus autores inter se discrcpare videntur: alii enim monstra duplicia e duorum germinum primarie separatorum fusione oriri, alii jam embryonum primordia coalita esse volunt. Autor theoriae duorum germinum primarie separatorum est D'Alton, ${ }^{\mathbf{1}}$ ) qui primus varietatem monstrorum duplicium e varia striarum primitivarum in uno blastodermate positione atque directione deduxit; quod etiam postea a Schulze Costioque acceptum atque comprobatum est. Illa oriundi ratio e duobus primarie separatis germinibus sine dubio valet in omnibus monstris duplicibus, quae omnino duplicia, modo leviter coalita, aut ventribus aut lateribus conjuncta apparent; neque minus valere videtur is in casibus, in quibus partes superfluae iis locis inveniuntur, quibus evolutae esse non possunt e primordiis illius embryonis, cui postea suspensae sunt, propriis; hic enim binum embryonum primordiorum unius embryonis primordia fere tota evanuisse, sumendum est.

1) De monstror: duplicium origine, Halis MDCCCIL. 
Sed D'Alton etiam ad ea monstra duplicia theoriam suam adhiberi vult, quorum columna vertebrarum atque medulla spinalis in altero fine (aut in anteriore aut in posteriore) duplex, in altero contra fine simplex invenitur; id enim fusione ante separatarum partium factum esse habet. Schulze ${ }^{1}$ ) contra talem primordiorum embryonum fusionem, ita ut pars simplex et aequalis fiat leges, physiologicas offendere habet; quod quidem nobis non videtur fieri, quum, si duae notae primitivae, sibi propinquiores, tamen separatae, obviam sibi succrescant, eas partes, quibus inter se contingant, (quae sunt igitur mediae) mutua crescendi vi tabescere, itaque corpus in fine simplex e binis primarie separatis embryonum primordiis exoriri posse, non difficile nobis videatur cogitatione comprehendere. Fusioni etiam favent, quae directo observavit Lereboullet2) in piscium arte seminatis ovulis: anno enim MDCCCLII in esocis lucii ovulo jam viginti quattuor horas post foecundationem duorum embryonum in communi vitello exstantium extremas caudas coalitas, atque postea paullatim plane coalescentes, chordas dorsales contra duplices permanentes vidit. Idem MDCCCLIII in aliis ovulis deformitatem jam tempore oriuntis notae primitivae exstetisse, atque postea evolutione modo mutatam esse observavit. Denique ei contigit, ut in tribus ovulis notae primitivae formationem directo observaret hoc modo: in cicatricula (bourrelet blastodermique) in conspectum venerunt duo tubercula prope sibi jacentia, quae producebantur ad efformandas notas primitivas (bandelet); tum sulci primitivi se ostendebant, qui eodem modo, ut notae primitivae directi erant. Laminae vertebrales, quum dividerentur, in partibus coalitis laminum exteriorum divisio bene processit, interiora contra valde ad coalescendum inclinabant atque postea re vera coaluerunt.

Unde sequitur, ut aliis in casibus embryonum primordia primarie separata semper tamen in uno vitello exstantia re vera coalescere possint, quod etiam D'Alton fieri sumsit; contra etiam aliis in casibus verisimile est, primordiodum embryonum coalitum multo ante factum esse; ita ut ille ante notae primitivae ortum jam perfectus, originem ducat e tempore, quo membrana germinativa formatur; quod etiam Schulze ${ }^{3}$ ) probavit, qui formationem notarum primitivarum primarie coalitarum e duobus primarie exstantibus vesiculis progerminativis deduxit. Illas duas sententias non sibi contrarias esse, sed simul valere posse patet, quum utraque re vera germina primarie duplicia fuisse velit.

Quae quum ita sint, monstra quibus extremitates superfluae suspensae sunt, secundum illam sententiam, quae duo primaria separata germina fuisse, vult, quod attinet ad ortum, in serie monstrorum duplicium collocanda essent; quod et idem

1) Virchow's Archiv, Be. 7, 1854. Ueber anomale Duplicität der Axenorgane.

Monatsschrift für Geburtskunde, Bd. 7. 1856. Ueber die Entstehung der Doppelmonstra.

2) Compt, rendus 1855 .

$\left.{ }^{3}\right)$ 1. c. 
D'Alton sumpsit; reque differrent a monstris duplieibus perfectis, nisi primordiorum unius embryonis tabe atque atrophia. Sed concedendum est, eorum ortum a duplicibus primordiis a priori deduci atque contendi non posse, quum fortasse, quae reliquiae alterius embryonis habeantur, tam exiguae appareant, ut dubitaretur, num re vera bina embryonum primordia perfecta exstetissent. Cui rei autem majorem lucem afferre posset serutatio anatomica diligenter instituta; hac enim fortasse alia alterius embryonis reliqua, quae leviorem inspectionem effugissent in conspectum venirent, gravia argumenta illius secundae sententiae, id est, ortus e duplicibus embryonum primordiis.

Serutationes autem anatomicae, ut omnino in teratologia, imprimis in casibus nostris, parum adhue perfecte atque diligenter factae sunt, quum exempla destruere cuntarentur. Monstra tamen anatomice non perscrutata, comparanda sunt, ut van Deen pracelare dicit, cum libris non dissectis. Ipse easus a Schneidero descriptus, jain supra commemoratus, atque ii casus, qui a D'Alton e collectione anatomica Halensi $^{1}$ ) afferuntur, non satis perfecte diligenterque perscrutati sunt, quum exempla prorsus delere non liceret. Quum igitur, ut jam supra dietum est, pateat, quo plures, partes, quae alterius embryonis esse possint, inveniantur, è propius esse vero, duplicia einbryonum primordia exstetisse, libentissime Prof. Panum hoo e sua collectione sumptum exemplum anatomice perserutandum mihi dedit: Erat enim pullus gallinaceus in quo exteriora duplicitatis signa, duae extremitates posteriores superfluae, duo ani exstabant; praeterea eaudae loco aliquid alae imperfectae simillimum se ostendebat. ${ }^{2}$ ) Primum igitur monstri figuram atque habitum diligentius describemus. Caput collum extremitatesque, quae pulli gallinacei propriae sunt, nornalem habebant figuram ac formam, legitimeque erant sita; neque corpus usque ad partem posteriorem quidquam anormale ostendebat. Mensurae pulli gallinacei, partibus superfuis praetermissis, hae erant:

In maxima extensione rostri apex a digiti longi apice distabat 171 millimetr.; rostrum longitudine erat 15 millimetr.; radix rostri a protuberantia occipitali distabat 23 millim., atque haec a rostri apice 39 millim.; capitis latitudo maxima erat 23 millim.; angulus maxillae inferioris a superiore sterni margine prominente distabat 60 millim.; antibrachium longum erat 24; atque carpus eadem longitudine; finis ejus inferior ab alae apice distabat 30 millim.; longitudo pennae nutantis maximae 62 mill.; ea femoris 25, cruris 34 millim.; calx ab articulo ossis metatarsi et digiti longi 25 , atque hic articulus a digiti longi apice 22 millim. distabat.

E quibus mensuris, tum e rostro crasso atque firmo, e pennarum nutantium non mediocri longitudine concludere licebat, pullum gallinaceum minimum per hebdomades tres vixisse.

1) De monstris, quibus extremitat. superfluae suspensae sunt, commentat. Halis MDCCCLIII. 2) Vid. tab. I fig, I et II. 
In extremo autem dorsi fine, fere in linea corporis media, duorum femorum extrema superiora, a protuberantia occipitali, collo extenso, 78 millim. distantia, e dorso fere sex millim. prominebant; ${ }^{1}$ ) femora ipsa, 25 millim. longa, minimis intervallis inter se distantia, deorsum directa, atque desuper in longitudinem 19 millim. tenui membrana inter se conjuncta erant. Medium inter illa fernorum extrema superiora spatium, ossiculum parvum, rotundum, 7 millin. longum, quod separatim moveri non poterat, obtinebat. ${ }^{2}$ ) Crura 31 millim. longa, rectis angulis cum femoribus, tamen articulis genuum mobilibus, conjuncta erant, atque iisdem fere rectis angulis in fronte apertis digrediebantur. ${ }^{3}$ ) Pedes normalem figuram ac formam ostendentes adducti erant angulis : acutis:

In utroque latere infra extrema femorum superiora erat anus; alter in sinistro latere, ${ }^{4}$ ) circiter 12 millim. infra illa femorum extrema situs, minor, atque minus ituberose proninens, rotunda apertura praeditus; alter in dextro latere, ${ }^{5}$ ) circiter 1.8 millim. infra femorum extrema, major, tuberis forman praebens, in hujus tuberis apice plicam habebat, in qua erat ostium. Duo ani a se invicem distabant 19 millim.

$\mathrm{Ab}$ is in utroque latere plicae membranacene secundum femora superflua et duas cruris partes producebantur, quasi pyramidis spatii latera formantes. 10 millim. supra anum dextrum, in dextri femoris superflui latere corporis pars exstabat, quae quidem, quod attinebat ad pennarum dispositionem, maximam alae imperfectae speciem praebebat; ${ }^{6}$ ) ossa tamen tactu non sentiebantur; ejus longitudo erat 30, atque ea pennae Iongissimae 25 millim.; eamdem directionem quani alae normales ostendebat; extremitates contra posteriores superfluae, ei normalium extremitatum contrariam directionem sequebantur. Illius partis, alae simillimae, aut extremitatum superfluarum motu simul anus dexter novebatur, unde has partes arctius inter se cohaeruisse, conclůdere licebat. Si jam normalium atque extremitatum superfluarum mensuras inter se camparamus, femora longitudine nihil, crura tria millimetra; distantiae calcium a metatarsorum articulis digitorum longorum 5 millim, atque eae horum articulorum a digitorum longorum apicibus 3 millim. inter se differunt; longitudo pennarum nutantium alarum normalium, ean pennarum ejus partis, quae ala simillima erat, $37 \mathrm{millim}$. superat. Unde sequitur, ut extremitates posteriorès superfluae vix normalibus in evolutione cederent; illa contra quae habebatur ala superflua ab alis normalibus evolutione longe superaretur.

Denique in abdominis parte inferiore, in linea media, a sterni superiore mair-

1) Vid. tabul. I, fig. I. et II. a.

$\left.{ }^{2}\right)$ Tabul. I. fig. I. et II. b.

3) Tabul. I. fig. II.

4) Tabul. I. fig. II. et taluul. II. fig: I. et II. c

${ }^{5}$ ) Tabul. I. fig. I. et II. et tab. II. fig. I. et II. d

6) Tabul. I. fig. I. et II. e. 
gine prominente 38 millim. distans foramen seu fissura, fere ex longo rotunda, paulIum infundibuliformis apparens, cujus diametros longissimus 12 millim., diametros internus 8, latitudo 5 millim. erat, se ostendebat. Centrum hujus foraminis ab ano dextro 19, a sinistro 10 millim. distabat. Ex hoc autem foramine intestina ad fascem pedunculatum conglutinata atque, ut visum est, cum fissurae lateribus coalita, 19 millim. propendebant; ${ }^{1}$ ) in fronte tamen et a tergo in cavum abdominale introspicere licebat.

Perscrutatione anatomica haec reperta sunt: paries abdominalis tenuissima, fere perspicua, nuda usque ad inferiorem sterni marginem; simili qualitate erant illae supra commemoratae plicae membranaceae, quae secundum femora et crura extremitatum posteriorum superfluarum producebantur. Intestina prolapsa non re vera cum fissurae lateribus coalita sed modo leviter agglutinata, quum facile reponerentur, se praebebant. Integumento abdominali discisso, illa ad fascem conglutinata intestina, quae prolapsa erant, et reliqua intestina et fere ubique cum peritoneo parietali et ex parte inter se leviter conglutinata apparebant; deinde, quod ex utroque ano fieri poterat, intestinis sub aqua inflatis, hoc spectaculum oculis subjectum est: ${ }^{2}$ ) cum ipsis anis saccus amplus, ${ }^{3}$ ) figura hominis ventriculo simillimus, cujus longitudo erat 15, diametros perpendicularis atque transversus 16 millim., commnnicavit. In dextro fine paullum constricto in intestinum ${ }^{4}$ ) 10 millim. longum, cujus diametros erat 7 millim., saccus ille transiit; atque hoc intestinum subito in quinque ramos exiit, quorum quattuor erant intestina coeca, ${ }^{5}$ ) in extremis nodosa, diametris fere aequalibus, longitudine tamen, 32, 40, 43, 45 millim., in quinto ${ }^{6}$ ) autern intestimum continuatum est. Totius intestini snb aqua evoluti longitudo a ventriculo usque ad saccum jam commemoratum erat circiter 525 millim. Reliqua viscera simplicia exstabant normalemque habitum praebebant. Vasa deferentia testium justo loco sitarum, atque renum, qui e partibus separatis constabant, ureteres normalem in modum in vestibulum sive saccun supra commemoratum introducebant. Deinde lateris dorsalis integumento resecto, columna vertebrarum in regione pelvina posteriore in dextrum latus declinantem se praebuit; quae tamen declinatio imprimis osse coccygis effecta est. In ossis coccygis extremo fine locum caudae illa jam supra commemorata pars alae simillima obtinebat; in qua autem, quamquam nulla ossa reperta sunt, quae de ejus natura lucem fortasse attulissent, tamen, quum et caudae locum obtineret, et pennae alae pennarum dispositionem ostenderent, eam caudam simul atque alam fuisse suspicari visum est. Proxime supra locum, quo insita erat corporis pars modo descripta, sub ipsa cute cum pulli

1) Tabul. I. fig. I. et II. f.

2) Tabul. II. fig. I.

3) Tabuil. II. fig. I. et III, g

4) Tabul. II. fig. I. et III. h.

$\left.{ }^{5}\right)$ Tabul. II. fig. I. i.

○) Tabul. II. fig. I. k. 
gallinacei corpore sola tela cellulosa laxa conjunctum corpusculum exstabat parruin, 4 millim. longum, et in margine majore 3 millim. latum, quod attinet ad massam, paene trigoni figuram praebens; facies ejus inferior paullum concava, facies superior paullum convexa; prope a inargine latiore parvula particula, 2 millim. longa, 1 millim. lata, plana simulque rotunda, ad massam principalem appressa se ostendebat, quae nudis jam oculis aspera apparuit; totum autem corpusculum simillimum erat alae imperfectae atque evolutione impeditae: Particula tenuissima ab hoc corpusculo abscissa, vitro paullum aucta, foveolas ostendebat clariores, quorum centra, adhibito vitro forti, corpuscula obscura parilliformia obtinebant; atque ab his, vitro fortiore auctis (ducenties) longae, solidae atque rigidae, undulatae fibrillae proficiscebantur, quarum nonnullae abruptae facies ostendebant angulosas; haud dubie autem pennae adhuc minime evolutae cognoscebantur. Alio loco illae fibrillae e foveolis, quorum centra obtinebant corpuscula papilliformia, prodibant. Praeterea in tela cellulosa organa obscura, prope sibi jacentia, glandularum sebacearum simillima conspiciebantur. Elementa ossea atque cartilaginea omnino non reperta sunt. Itaque illud corpusculum alam imperfectam minimeque evolutam fuisse, non sine veritatis specie nobis esse videtur, ita ut papillas commemoratas pennarum papillas, atque illas fibrillas pennae (Fedevfahne) elementa habeamus.

Illud autem jam supra commenoratum ossiculum firmum ac solidum, cum quo femorum extrema superiora conjuncta erant, manifesto constabat ex osse sacro atque osse coccygis coalitis. Ea, quae os coccygis repraesentabat, pars 7 millim. longa, acuminata, angulo recto cum reliqua ossis coaliti parte latiore atque crassiore, 8 millim. longa, quae anteo sub cute abdita fuerat conjuncta erat. In hujus ossis extremo superiore vertebra mobilis adhuc cartilaginea exstabat. Hae omnes partes sola tela cellulosa cum pullo gallinaceo conjunctae erant. Infra angulum quem formabat illud ex osse sacro et osse coccygis coalitum ossiculum, a lateribus tela cellulosa firme conjuncta cum eo, in tela cellulosa cutis occulta, duo ossa ${ }^{\mathbf{l}}$ ) plana atque pertenuia, fere trigonorum figuras praebentia exstabant; cujusque ossis alterum extremum ${ }^{2}$ ) deorsum in pulli gallinacei telam cellulosam in cacuminis finem desiit; altera extrema latiora angulo acuto adversus os ex osse sacro et osse coccygis coalitum atque femorum extrema superiora inter se concurrebant, in duos ramos, foramine ${ }^{3}$ ) separatos diffindebantur. Alter ramus inter femorum extrema superiora adversus angulum, quo os sacrum et os coccygis inter se conjuncta erant, procurrens hoc loco congrediebatur cum alterius lateris ramo ita ut quodammodo decussarentur. Alter autem ramus cum superiore, latiore ossis sacri parte conjunctus erat.
1) Vid, tab. Il. fig. IV. I
2) Ibid. m.
3) Ibid. n. 
Margines in os sacrum imperfectum atque femorum extrema spectantes, sive bases trans foramen illud supra commemoratum mensi longitudine erant 3 millim., reliqui margines a basi usque ad apices sive symphyseos extrema, dextri ossis 15 et 12 millim., ii, ossis sinistri 11 et 10 millim. longi erant, ita ut os dextrum mannitudine paullum superaret sinistrum. Symphyseos extrema adhuc erant eartilaginea.

Ossa igitur modo descripta una cum illo osse ex osse sacro et osse coccygis constante, pelvem, tamen pathologice formatam repraesentabant.

Haec pelvis eamdem pelvis normalis pulli gallinacei directionem sceuebatur; unde sequitur, ut extremitates posteriores superfluae, quae jan supra pulli gallinacei extremitatibus nurmalibus directione oppositae commenoratae sunt, quod attinebat ad pelvis earum propriae directionem, distortae essent.

$\mathrm{Ab}$ utroque pelvis superfluac lątere musculus pertenuis, cujus elementa vitro aucta, fibras transverse striatas se praebuerunt, ad duos anos decurrebant.

Denique in angulo, quem os sacrum et os coccygis formabant, eo loco, quo illi ossis coxae rami utrimque concurrebant, femora superflua, non capitibus instructa, sola tela cellulosa mobiliter insita erant. Cutis pertenuis, qua extrernitates posteriores superfluae erant indutae, vitro aucta, praeter epidermidem reticulatos telae cellulosae fasciculos, et ducenties quadragies aucta, nonnullos pertenues, claros, ramificatos tubulos ostendebat, de quibus in dubio erat, num vasa capillaria an fibrae nerveae habendi essent. Sub cute inter femora practer telam cellulosam ubique exstantem tela sufflava conspiciebatur, quae microscopio adhibito, fibras ostendebat rigidas, in memoriam redigentes fibras musculares, nec tamen in longum nec in transversum striatas. Reliqua pedum superfluorum ossa normalia se habebant.

Perscrutatione igitur anatomica modo instituta conjectura supra facta, fortasse alterius embryonis partes, quae leviorem inspectionem effugerent, exstare, omnino comprobata est. Itaque ad ultimum colligentes, quae in nostro exemplo superflua reperta sunt: 1) columnae yertebrarum rudimentum ex osse sacro, osse coccygis, vertebra unica constans, 2) reliqua pelvis ossa atque extremitates posteriores, 3) corpusculum haud dubie ala imperfectum sumendum, atque alia pars hand dubie ex ala et cauda composita, 4) anus, 5) duo intestina coeca, quum quattuor intestinorum coecorum, quae reperta sunt, duo sola galli normaliter propria sint; denique 6) saccus ille, (sive vestibulum) qui, quum communicaret cum utroque ano, haud dubie e duobus saccis erat compositus; in nostro casu duo embryonum primordia exstetisse certum nobis est. Alterius embryonis primordiorum superior pars, alis imperfectis, quae in pulli gallinacei corporis parte posteriore remanserunt, exceptis, prorsus evanuit; inferior contra pars imperfecte evoluta est.

Justissime igitur exemplum descriptum in serie monstrorum duplicium verorum collocandum nobis videtur. 
Quae quum ita sint, etiam in aliis casibus, fortasse in plurimis nisi in omnibus, in quibus extremitates superfluae occurrunt, alterius embryonis reliqua etiamsi exigua atque jam in evolutionis initiis impedita, perscrutatione anatomica diligenter facta, exstare, sumendum est; quare, ut ejusmodi perscrutationes anatomicae saepius instituerentur, optabilis esset.

Jam antem, quod attinet ad illorum monstrorum ortum atque evolutionem innprimis tres existunt quaestiones:

I. Unde facta sit atrophia alterius embryonis primordiorum?

Variissimi atrophiae gradus a perfectissimis monstris duplicibus aequalibus usque ad monstra, quae dicuntur parasitica, in quibus numerantur monstra nostra extremitatibus superfluis praedita, occurrunt. Aut causae atrophiae erant diversae, aut eaedem causae variam habuerunt vim ad effuiendam atrophiam; aut atrophia duobus his causis facta est; sed hae res adhuc luce carent. Hoc quidem certissimum videtur, in casibus nostris, in quibus alterius embryonis reliqua inveniuntur, jam primo evolutionis tempore causas eam impedientes valuisse atque has in nutritione non sufficiente quaerendas esse. Quomodo autem fit nutritio non sufficiens? Hic Prof. Panum sententia maxime valere videtur: idem enim in monstris uostris circulationem similem in modum effici, atque eorum evolutionem similem in modum ex ea pendere, quem Dr. Claudius ${ }^{1}$ ) in acardiacis pracelare excellenterque demonstravit, verisimillimum habet. Quum enim in his vasorum funiculi umbilicalis communicatio alterius embryonis evolutionem impediat, ratione omnino physica, quam Dr. Claudius explicavit, secundum Prot. Panum sententiam in monstris nostris duplicibus eaedem circulationis rationes, sed tempore longe priore, fortasse eo tempore, quo vasa evolvuntur, quaerendae essent. Si enim quod in monstris nostris, quorum primordia erant duplicia, verisimillimum est, duo circulationis centra evolvuntur, vasa propagata anastomosi conjungerentur itaque sanguis utriusque centri sibi ubviam duceretur, quare idem, quod vasorum umbilicalium communicatione in acardiacis, efficeretur; ita ut alterius embryonis centrum desineret centrum esse atque alterius embryonis vasa in altero modo continuarentur. Itaque in altero embryone nutritio fieret minus perfecta; in aliis ejus partibus tamen imperfectior quan in aliis; quin etiam in aliis partibus nutritio adeo impediretur ut atrophia corriperentur atque paullatim evanescerent; quod quidem in nostri generis monstris factum esse videtur, quum in is praeter paucas perfecte evolutas partes, vestigia modo secundi embryonis exigua reperiantur. E vario autem modo, quo utriusque embryonis vasa anastomosi conjungerentur, varii atrophiae gradus atque habitus monstrorum duplicium parasiticorum deducerentur.

II. Quo modo fiat primordiorum embryonum cealitus?

Coalitio ipsa perpauris in casibus directo observata est, ut a Lereboullet 2 ) in

1) Die Entwicklung der herzlosen Missgeburten. Kiel 1859 .

2) Pag. 3 et Compt. rend. 1855 . 
piscium ovulis. Semper unbryonum primordia in uno exstabant vitello atque semper primo evolutionis tempore facta est coalitio. Quomodo haec coalitio intelligitur? Hic sequerer Prof. Panum, qui secundum quaestiones suas de avium monstrorum ortu, hanc sententiam de coalitione exponit: si duo in uno vitello exstantes cellularum acervuli sibi occrescunt, denique inter se contingent; vires plasticae sibi obluctantes se invicem compensando tollentur; cellularum autem strata, illis viribus sibi obluctantibus pressa, atrophia correpta evanescent itaque denique partium duarum coalitio fieri poterit. Hac ratione primarii cellularum embryonum acervuli coalescere possunt, sive columnarum vertebrarum, sive aliorum organorum primordia utrimque angulo inter se concurrunt. Monstrorum autem duplicium varietas, ut D'Alton et postea Schulze et Coste sumpserunt, e primordiorum embryonum positione atque directione deducenda esset. In Monstris autem duplicibus etiam atrophia atque tabes alterius embryonis primordiorum ad monstri duplicis formam ac figuram maxime valent, partim fortasse imparibus viribus plasticis sibi obluctantibus, partim fortasse illa supra commemorata circulationis ratione; ita ut nonnullis in casibus monstri duplicis evolutionem et tabe et atrophia alterius embryonis mutatam atque moderatam sequi atque intelligere, difficillimum sit. De hac autem re excellenter edocent Valentini ${ }^{1}$ ) in piscium émbryonibus observationes.

III. Qua re avium monstrorum duplicium pro numero major crebritas intelligatur?

In omnibus collectionibus atque museis avium monstra duplicia, imprimis ea, de quibus modo scripsimus creberrima occurrunt; quam ob rem avium ova longe crebrius, quam exempli causa mammalium ovula ea qualitate, uae monstrorum duplicium ortui faveat, esse viderentur. Prof. Panum tamen quaestionibus suis, quae proxime in libro edentur, reperiit, in avium ovis, quorum evolutio impedita est, monstra simplicia creberrime inesse. Haec autem monstra simplicia raro modo e testa se expedire possunt, ita ut observatores facile effugerent, eamque ob causam rara in collectionibus asservantur. Monstra duplicia contra, imprimis ea, quae extremitatibus superfluis praedita sunt, plerumque facile e testa se expedire possunt itaque in conspectum venientes in musea deferuntur. Prof. Panum igitur avium monstra simplicia in ovis inquisita revera longe crebiora exstare quam monstra duplicia, affirmat, atque monstrorum duplicium crebritatem ab ea monstrorum simplicium in avibus et mammalibus longe differre, valde dubitat.

1) Vierordt's Archiv für physiologische Heilkunde. 1851. pag. 1 - 40. 


\section{T H E S E S.}

I. Syphilisatio a priori tutamentum syphilidis rejicienda est.

II. Partus praematurus optime efficitur vaginam aqua tepida saepius irrigando.

III. Diaeta moderata optimo remedio utendum est in vitiis cordis organicis. 



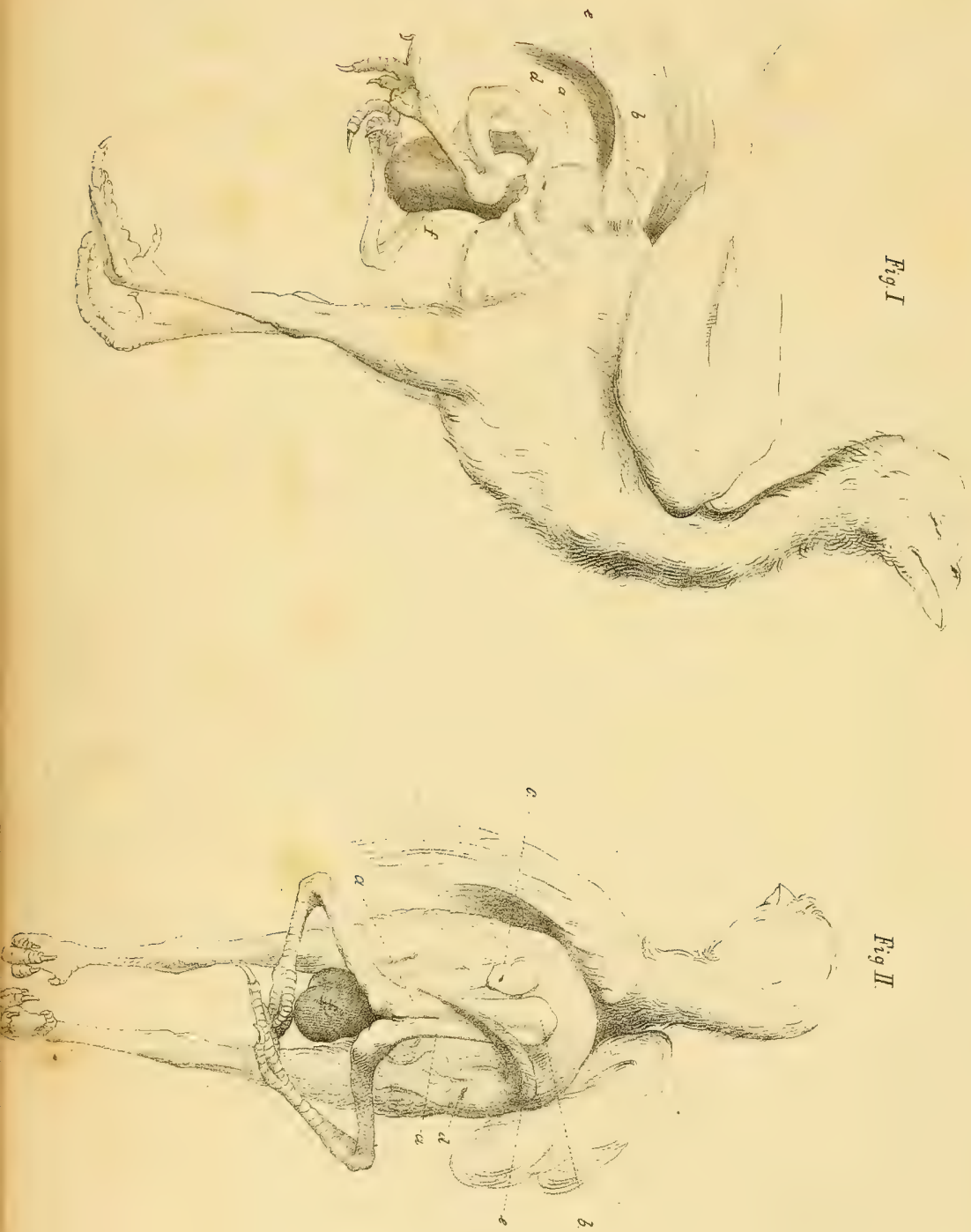


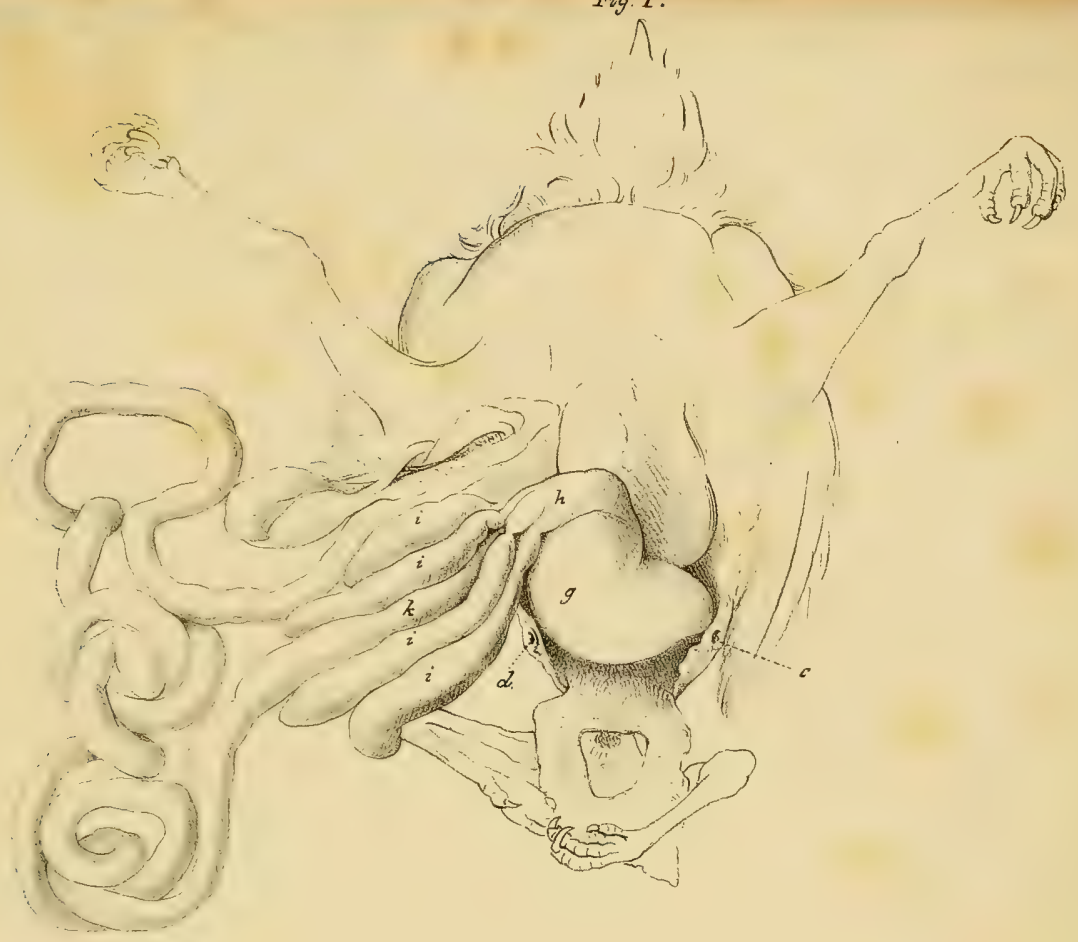

Fig.II.

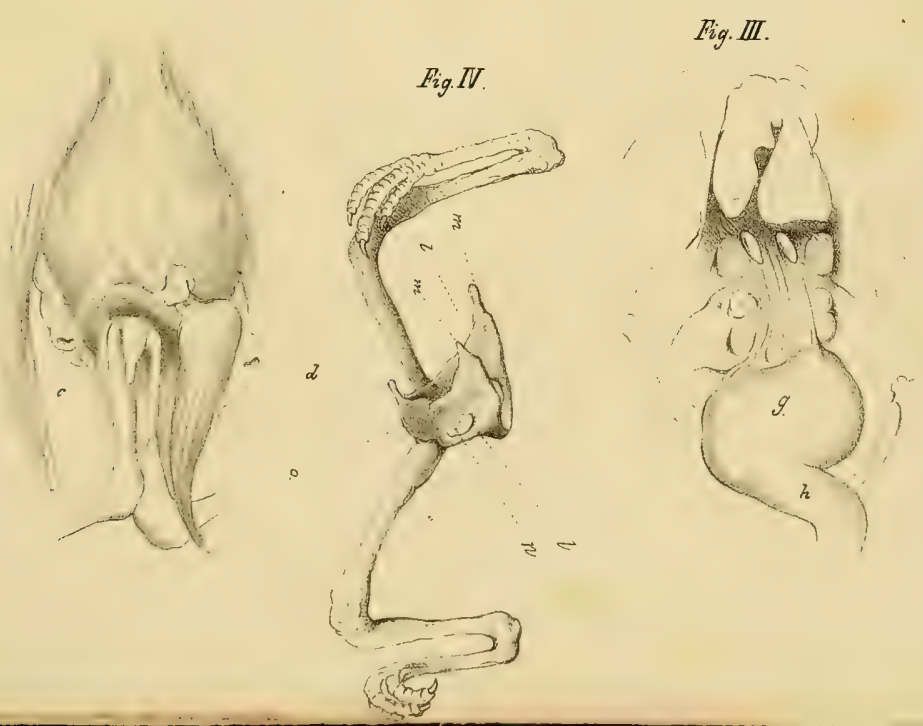









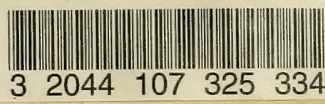




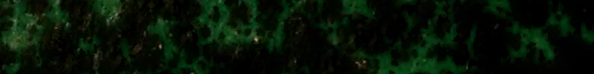

6.

2.

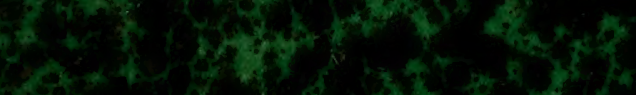

(5)

然

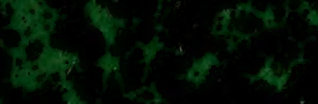

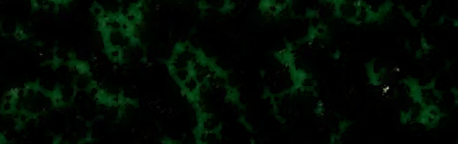

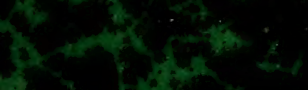

$\log ^{2}+2 x^{2}-12$

$x^{2}-3 x+2 x$

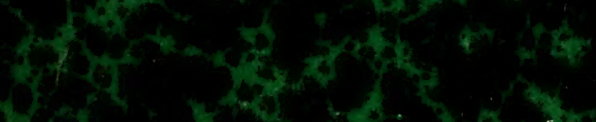

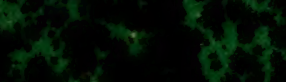

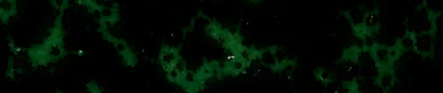

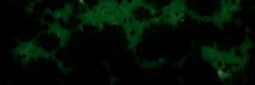

7.

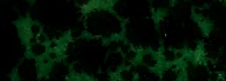
(4)
Hexis $x+3$
$+3$
is.

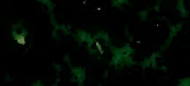

6.

$x^{2}$

- $3 x^{2}+x^{2}$

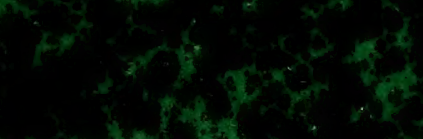

Cy

(3) Wy

C.t.

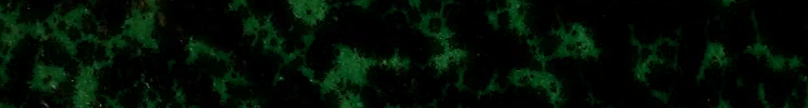

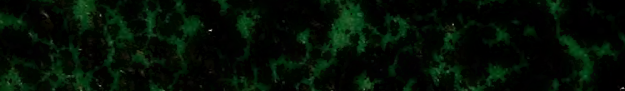

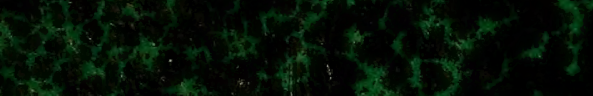

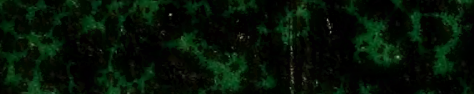

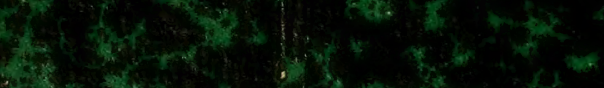
We 